\title{
Germanica
}

\section{Le repas africain dans le roman d'Ulrike Draesner Sieben Sprünge vom Rand der Welt (2014)}

The African meal in Ulrike Draesner's novel Sieben Sprünge vom Rand der Welt (2014)

Das afrikanische Essen in Ulrike Draesners Roman Sieben Sprünge vom Rand der Welt (2014)

Joëlle Stoupy

\section{(QpenEdition}

\section{Journals}

Édition électronique

URL : https://journals.openedition.org/germanica/3055

DOI : 10.4000/germanica.3055

ISSN : 2107-0784

\section{Éditeur}

Université de Lille

Édition imprimée

Date de publication : 30 décembre 2015

Pagination : 127-137

ISBN : 9782913857360

ISSN : 0984-2632

\section{Référence électronique}

Joëlle Stoupy, «Le repas africain dans le roman d'Ulrike Draesner Sieben Sprünge vom Rand der Welt

(2014) », Germanica [En ligne], 57 | 2015, mis en ligne le 30 décembre 2017, consulté le 16 août 2022

URL : http://journals.openedition.org/germanica/3055; DOI : https://doi.org/10.4000/germanica.3055 


\title{
Le repas africain dans le roman d'Ulrike Draesner Sieben Sprünge vom Rand der Welt (2014)
}

\author{
Joëlle STOUPY \\ Université du Littoral-Côte d'Opale
}

Dans son roman Mitgift (2002), Ulrike Draesner a consacré la partie intitulée « Essen », à l'analyse du rapport à la nourriture de son personnage principal Aloe. Elle y a observé les différentes phases de l'anorexie de ce personnage. Son dernier roman, publié en 2014 et qui a pour titre Sieben Sprünge vom Rand der Welt raconte, lui, sur plusieurs générations, l'histoire de deux familles, la famille allemande Grolmann, qui a dû quitter en 1945 la ville d'Oels en Silésie, et la famille polonaise Nienaltowski qui, elle, a dû laisser derrière elle, à la même époque, la Pologne orientale pour s'installer à Wrocław (Breslau). Ce roman polyphonique qui fait parler à tour de rôle les membres des différentes générations met en scène la guerre, l'exode et l'expulsion, mais se situe aussi à notre époque. Parmi la génération qui n'a pas directement connu la guerre, on trouve le personnage de Simone Grolmann, éthologue et celui de Boris Nienalt (il ne se fait pas appeler Nienaltowski), psychologue, dont le sujet d'étude porte sur les traumatismes dus à la Seconde Guerre mondiale. La relation entre Simone et Boris commence stricto sensu par un repas dans un restaurant africain. Ce repas va peu à peu 
servir de cadre à un échange très intime sur des sujets qui sont au cœur même du roman : les souvenirs et émotions transmises d'une génération à l'autre suite à des expériences traumatisantes. C'est pendant ce repas africain que Boris parle à Simone pour la première fois des recherches menées par les Américains sur la post-mémoire et que Simone se confie sans retenue à Boris sans vraiment le connaître.

Simone Grolmann ne connaît de Boris Nienalt tout d'abord que sa voix à la radio. Elle est en premier lieu interpelée par cette voix mélodieuse, sonore et douce à la fois. Mais aussi et surtout par les propos de Boris sur la génération née entre 1927 et 1940 qui a connu la guerre pendant l'enfance ou l'adolescence. Si elle est si attentive à ses propos, c'est que son père Eustachius Grolmann, avec qui elle entretient une relation plus ou moins conflictuelle, a connu, alors qu'il avait 14 ans, l'exode qui a fait de lui un être singulier. Les propos de Boris font tout de suite naître en elle le souvenir de la maison de ses grands-parents paternels où l'on chantait des chansons qu'elle connaissait par cœur sans toutefois les comprendre. On y parlait aussi « de la faim, des coups, des pertes, du pays natal »1. Si le mari de Simone Grolmann, Johnny, veut tirer un trait sur ce passé et ne veut plus en entendre parler, il est évident que leur relation en a souffert, qu'elle est maintenant sur la fin et que Boris, lui, sera d'autant plus proche de Simone qu'il s'intéresse à ce passé, qu'il a, malgré certaines différences évidentes, une histoire familiale similaire et qu'il fait ressurgir en elle cette contrée « engloutie», la Silésie. Simone Grolmann entend pour la deuxième fois la voix de Boris Nienalt à la radio alors qu'elle est dans sa cuisine en train de confectionner un gâteau. Pour la seconde fois, les paroles de Boris la saisissent. Ces propos vont faire que Simone Grolmann feront qu'elle voudra en savoir plus sur les « enfants d'Hitler $»^{2}$ et elle contactera Boris par courriel pour lui confier une mission concernant son père. En apprenant, en effet, que son père a emprunté 430000 euros pour un projet qu'elle ignore - son père, professeur émérite en éthologie, admirateur comme elle des grands singes, en a deux chez lui qu'il détient illégalement et pour qui il veut construire une sorte de paradis -, elle a l'idée de faire intervenir un psychologue à qui Eustachius pourra dire la vérité.

Quant à Boris Nienalt il apparait au lecteur pour la première fois dans le chapitre qui lui est consacré, dans le bistrot qui se trouve près de son cabinet et dans lequel il se rend plus fréquemment depuis qu'il est

1. - «Gesprochen wurde über Dinge, die außerhalb der Wohnung nicht wahr sein durften oder sollten, den Hunger, die Schläge, die Verluste, die Heimat» (Ulrike Draesner: Sieben Sprünge vom Rand der Welt. München, Luchterhand Literaturverlag, 2014, p. 15).

2. - «Hitlers Kinder hätten Spuren auch in den Psychen ihrer Söhne, Töchter und Enkel hinterlassen» (ibid., p. 22). 
séparé de sa femme Antonia. À la troisième bière, il évoque devant son ami Marek le courriel qu'il a reçu de Simone Grolmann - un message qu'il juge " incroyable », car cette inconnue lui a déjà avoué aimer sa voix. Une fois dans son cabinet qui lui sert d'appartement, au milieu des caisses de déménagement, Boris, éprouvant du dépit face à sa situation personnelle, se saoule tout en pensant à Simone Grolmann et à son père Eustachius :

Tochter, Vater, ich. In der Küche warf ich mir drei Salzbrezeln auf einmal in den aufgerissenen Mund. Spülte mein Glas. Es glänzte perfekt, noch während es fiel ${ }^{3}$.

Sa minuscule cuisine dans laquelle il cherche ensuite une bouteille de wodka n'a jamais servi à cuisiner. Elle semble avoir pour fonction de conserver l'alcool qui lui sert à oublier son mal-être - un état dû à sa séparation, mais aussi et surtout à ses origines (« Ich war hier nicht aufgewachsen, lebte hier bloß $»^{4}$ ) - il a grandi en Pologne et a un père allemand. On le verra attablé pour la première fois avec Simone Grolmann - un moment qui va amorcer leur relation. C'est parce qu'il retrouve en Simone son histoire personnelle qu'il sera entre autres attiré par elle :

Die Frau passte optimal »zu meinem Skript«, ihre Herkunftsgeschichte zog mich an, auch in ihrem Leben fehlte ein Vater, auch sie war das Kind eines Flüchtlingskindes ${ }^{5}$.

Avant de mettre en scène le repas africain, l'auteur nous fait assister à une conférence de Simone Grolmann sur les grands singes et leur rapport à la nourriture au cours de laquelle Boris voit Simone pour la première fois en chair et en os. Simone y évoque avec brio et humour une expérience faite sur les chimpanzés. On y voit un premier chimpanzé obtenir un tas de légumes après avoir tiré sur une corde reliée à une table à roulettes. L'expérience fonctionne, mais en présence d'un autre chimpanzé, le premier n'agit plus, car il a peur de devoir se battre. Si par contre les portions de légumes sont distinctes, les chimpanzés coopèrent :

Die Professorin zeigte den bekannten Rolltisch. An jedem seiner Enden prunkte ein fein geschnittenes Hügelchen Grünzeug, vor jedem kaute ein zufriedener Schimpanse ${ }^{6}$.

\footnotetext{
3. - Ibid., p. 74.

4. - Ibid., p. 75.

5. - Ibid., p. 101.

6. - Ibid., p. 120.
} 
Le lecteur a encore en mémoire l'image des chimpanzés satisfaits assis devant leur tas de légumes quand débute, dans le chapitre consacré à Boris Nienalt, le repas africain. Nous apprenons dans ce chapitre que Boris a écrit à Simone tout de suite après la conférence. Parce qu'il voulait lui faire comprendre ses véritables motivations, l'intérêt qu'il lui porte, il s'applique dans son courriel à choisir les mots qui lui semblent appropriés pour n'être ni « trop proche, [ni] trop importun, [ni] trop distant $»^{7}$. Le courriel que Boris finalement écrit à Simone est si explicite sur les sentiments qui l'animent qu'elle accepte vraisemblablement ce repas déjà comme un premier tête-à-tête amoureux. Il importe alors à Boris d'être celui qui invite, celui qui paiera l'addition, une façon de montrer à Simone Grolmann, qui dans la séquence précédente l'a impressionné par son savoir et sa prestance, qu'il veut à présent mener le jeu. Simone n'est pas heurtée par cette proposition, mais s'en réjouit, montrant ainsi qu'elle accepte tacitement l'intérêt de Boris pour elle. Après une rencontre manquée à l'institut où travaille Simone, Boris est heureux de la compagnie de cette femme brillante qu'il avait appelée, en voyant une photo d'elle sur Internet, " une Catherine Deneuve de l'éthologie ${ }^{8}$. C'est au restaurant la beauté et la sérénité de Simone qui fascinent Boris.

Ce restaurant exotique orné de masques africains, où l'air chaud, empli d'épices et de musique ethnique, contraste avec le temps au dehors en plein mois de mars, met en scène les deux personnages dans une situation tout d'abord cocasse. On les voit assis inconfortablement sur des nattes de raphia à une table, et ils ont dû se rapprocher involontairement, car le jeune serveur, les prenant vraisemblablement déjà pour un couple d'amoureux, leur a apporté une seule carte. Les mets qui sont servis dans ce restaurant sont aussi étonnants et prêtent à sourire. Les galettes qu'ils doivent enrouler eux-mêmes autour de légumes ou de morceaux de poulet sont si grandes que les assiettes n'y suffisent pas et elles en débordent. Elles sont « grandes comme des roues de voiture » ${ }^{9}$. Simone, elle, reste davantage maîtresse de la situation tandis que Boris se débat avec les herbes pendant de sa galette. Ces passages drôles alternent avec des moments d'intense sérieux qui parlent de questions graves comme des traumatismes de l'enfance. En effet, après avoir discuté comme des adolescents de sexualité humaine et animale et échangé des idées plus ou moins farfelues sur la virilité et la fidélité, Simone évoque le cas de son père, et leur conversation prend alors un tour plus sérieux en abordant le thème de la fuite. Le sujet de l'exode est introduit par la phrase

7. - «Ich hatte ihr nach dem Vortrag geschrieben, eine kleine Ewigkeit an den paar Zeilen gefeilt, nicht zu nah, zu aufdringlich, zu distanziert [...]» (Ibid., p. 124).

8. — «[...] eine Catherine Deneuve der Verhaltensforschung [...]» (Ibid., p. 71).

9. - «[...] groß wie Wagenräder» (Ibid., p. 127). 
d'Eustachius Grolmann - «Als alter Mann werde ich glücklich sein » - que celui-ci a répété de nombreuses fois devant sa fille et qu'il a aussi confié à Boris comme une sorte de phrase-clé10, oubliant qu'à 83 ans, il est déjà vieux. Mais cette phrase lui sert toujours de consolation après qu'il a vécu l'expérience traumatisante de la fuite de Silésie. Le souhait qu'exprime Simone pendant ce repas d'avoir un père « normal » ayant une vieillesse «normale » semble ainsi irréaliste quand on considère qu'elle-même, qui n'a pas vécu directement ce traumatisme, souffre de ce passé. Elle apprend à Boris dans ce passage que ses rêves sont hantés par les images que son père a fait naître en elle par son comportement, ses non-dits et ses récits de la fuite de Silésie. Parce qu'il a dû traverser la forêt de Breslau par $-21^{\circ}$, dans la nuit du 19 au 20 janvier 1945, avec sa mère Lilly et son frère handicapé Emil, dans une neige toujours hostile, Simone aujourd'hui vit cette angoisse d'autrefois et a peur de la neige, comme elle le confie à Boris pendant le repas africain. Cette confidence entraîne celle de Boris et elle les conduit, sous le coup de l'émotion, à se tutoyer au milieu du repas. Boris, connaît aussi les peurs d'Halka, sa mère :

Meine Mutter, sagte ich, habe in ihrem ersten Jahr in Wrocław mit einer Pistole unterm Kissen geschlafen, bei verrammelten Türen und zugenagelten Fenstern. Später habe sie Vorhänge und Jalousien in ihren Wohnungen nicht mehr ertragen, ja, die Fenster sogar im Winter aufgerissen. Wie ich. Der ich ihre Angst, ihre Abneigung übernommen hätte, um sie ihr leichter zu machen. Eine Kindertat. Ganz Kindeskind ${ }^{11}$.

Après ces révélations, leur rencontre apparaît à Simone subitement comme une chance inespérée, un « coup au but $»^{12}$, tandis que Boris en oublie ses incertitudes pour sourire à Simone en sentant pour la première fois depuis longtemps son cœur battre. Peu de temps auparavant, la présence de cette femme qui lui semblait à la fois proche et distante avait provoqué en lui une sorte de dédoublement, maintenant disparu devant la force des sentiments qui l'anime. Il est alors à même de lui confier son carnet de notes et de lui parler des recherches sur les traumatismes dus à la Seconde Guerre mondiale sur lesquelles il s'appuie.

Boris se base, en effet, dans ses travaux sur les recherches de certains auteurs contemporains d'expression anglo-américaine comme Marianne Hirsch, Eva Hoffman ou Lisa Appignanesi. Ces trois noms sont nommés par Boris au cours du repas africain. Comme nous l'apprend Ulrike Draesner dans son site consacré à son roman, Marianne

10. - Cf. www.der-siebte-sprung.de/als-alter-mann-werde-ich-gluecklich-sein/

11. - Ulrike Draesner: Sieben Sprünge vom Rand der Welt, op. cit., p. 129.

12. - «Dass ich dich aussuchte, war wohl ein Volltreffer» (Ibid.). 
Hirsch a été la première à parler de post-mémoire (postmemory) au début des années 1990 dans un article consacré à la bande dessinée Maus d'Art Spiegelman. Marianne Hirsch, née peu après la Seconde Guerre mondiale en Roumanie, s'intéresse aux enfants de victimes de traumatismes, notamment de l'Holocauste. Le terme de post-mémoire est utilisé par Marianne Hirsch, nous confie Stéphanie Bellemare-Page dans « La littérature au temps de la post-mémoire : écriture et résilience chez Andreï Makine »13, « pour désigner l'expérience des personnes ayant grandi entourées de récits de survivants de la Deuxième Guerre mondiale. [...] [P]our eux, la médiation avec le passé ne s'effectue pas par l'entremise du souvenir mais par l'entremise de l'imaginaire. Leur perception de la guerre s'appuie donc sur des récits et non pas sur une expérience vécue».

Dans son ouvrage The Generation of Postmemory. Writing and Visual Culture After the Holocaust (2012), où elle développe le concept de post-mémoire, Marianne Hirsch s'étonne de pouvoir évoquer des moments précis de la vie de ses parents pendant la guerre, des moments qu'elle n'a pas vécus, tandis que ses propres souvenirs de jeunesse sont moins présents :

Why could I describe the streets, residences, and schools of preWorld War I Czernowitz and interwar Cernăuti, where they grew up, the corner where they evaded deportation, the knock on the door in the middle of the night, the house in the ghetto where they waited for deportation waivers - all moments and sites that preceded my birth when I had lost the textures, smells, and tastes of the urban and domestic spaces in Bucharest where I spent my own early life? ${ }^{14}$

La force et présence obsédante des récits de la génération ayant vécu des traumatismes est telle, pense Marianne Hirsch, que ceux qui y sont confrontés sont menacés de perdre leur propre histoire ${ }^{15}$. Marianne Hirsch évoque aussi les recherches d'Eva Hoffman, née à Cracovie en Pologne et dont les parents ont survécu à la guerre en se cachant, et elle met en avant le concept de broken refrains qu'Ulrike Draesner met dans la bouche de son personnage Boris Nienalt pendant le repas africain. Eva Hoffman a, elle aussi, été confrontée au chaos émotionnel dû aux événements traumatisants vécus par ses parents :

13. - www.erudit.org/rvue/etudlitt/2006/v38/n1/014821ar.htlm.

14. - Marianne Hirsch: The Generation of Postmemory. Writing and Visual Culture After the Holocaust, New York, Columbia University Press, 2012, p. 4.

15. - «To grow up with overwhelming inherited memories, to be dominated by narratives that preceded one's birth or one's consciousness, is to risk having one's own life stories displaced, even evacuated, by our ancestors» (Ibid., p. 5). 
The memories - not memories but emanations - of wartime experiences kept erupting in flashes of imagery; in abrupt but broken refrains ${ }^{16}$.

Le troisième auteur évoqué pendant le repas africain est Lisa Appignanesi, née Elżbieta Borenszteyn à Łódź en Pologne, en 1946. Ses parents étaient des survivants de l'Holocauste. Le personnage de Boris Nienalt s'appuie sur deux concepts tirés de l'œuvre de Lisa Appignanesi, le concept de transgenerational haunting et celui de memories cascading through lives. Dans son œuvre Losing the Dead (2013) qui raconte l'expérience de ses parents juifs polonais pendant la Seconde Guerre mondiale, Lisa Appignanesi écrit à propos de son livre :

So this is a journey into my parents' past - into that foreign country they carried within themselves, which was also the country of war. The psychological tropes, the ways of confronting and filtering experience, which structured their lives grew largely out of that war and subsequent immigration. I suspect they passed these patterns on to my brother and me, as surely as they passed on their genes and with as little choosing. Understanding this transgenerational haunting is part of the journey - and perhaps in a century where migration, forced or chosen, is the norm, it is its most common part. Memory, like history, is uncontrollable. It manifests itself in unruly ways. It cascades through the generations in a series of misplaced fears, mysterious wounds, odd habits. The child inhabits the texture of these fears and habits, without knowing they are memory ${ }^{17}$.

Sur le site Internet qu'elle consacre à son roman, Ulrike Draesner s'arrête longuement sur le concept de Memories Cascading qu'utilise Lisa Appignanesi et dont parle Boris Nienalt pendant le repas africain. Elle y écrit :

Was Kinder unbewusst von Eltern übernehmen, betrifft nicht nur Gefühle, Gefühlsstrukturen und Habitus, sondern kann Träume und Ängste generieren und zur Entwicklung körperlicher Symptome führen. Manchmal, wenn ich von meinem Romanprojekt erzählte, antworteten mir Freunde oder Bekannten, dass sie manchmal Träume träumten, die sie dem Leben eines ihrer Elternteile zuordnen könnten. Begleitet von irrationalen Ängsten vor Hunger, Schnee oder Regen [...] Das Phänomen ist bekannt, doch wenig erklärt. Memories cascading - Erinnerungen, die wie Wasser, mit Druck, durch den Kopf rauschen, dabei fallen und verharren, wieder fallen, scheint mir ein gutes Bild

16. - Ibid., p. 31 .

17. - Lisa Appignanesi : Losing the Dead, London, Chatto \& Windus, 1999. 
dafür. Ein unwillkürlicher Prozess - Fall und Weiterfall des Falles, Flüchtling gewesen zu sein ${ }^{18}$.

C'est en pensant à ce phénomène qu'Ulrike Draesner fait dire à son personnage, Simone Grolmann, pendant le repas africain :

« Manchmal träume ich seine Bilder. Finden Sie das komisch?» / Sie schluckte: «Und ich habe Angst vor Schnee ${ }^{19}$.

Le premier chapitre consacré à Simone Grolmann nous montre quelles « images» hantent parfois ses rêves. Ce passage est intitulé, sur le site Internet consacré à ce roman, « Simone und die Träume aus einem anderen Leben ». On y voit un jeune garçon transi de froid, qui s'avère être le jeune Eustachius, à la recherche de pain pendant l'exode. Dans les images qui la hantent, Simone voit un gymnase rempli de tables où l'on a déposé des coupes de cristal, des vases, des carafes, des verres à champagne et autres objets de valeur disposés là en attendant l'arrivée des Soviétiques et à leur intention, et elle voit aussi parmi ces tables richement décorés, mais désespérément vides de nourriture, le jeune Eustachius rongé par la faim.

Parmi les événements traumatisants dont parlent Boris et Simone pendant le repas africain, on trouve, en effet, le thème récurrent de la faim pour les générations qui ont connu la guerre et la fuite. Eustachius se rappelle par exemple que pendant l'exode, il était presque toujours assis sur sa valise « avec l'estomac qui gargouillait ${ }^{20}$ de faim. Le quotidien des personnes expulsées est fait de faim, d'attente, de marches harassantes, de froid ${ }^{21}$. La faim est si omniprésente que tout la rappelle. La nuit, on se réveille après avoir rêvé de pain. La nourriture devient pendant ces temps difficiles une monnaie d'échange. Eustachius échange sur le marché noir contre de la nourriture la petite voiture construite pour son frère handicapé, maintenant disparu. Les vêtements d'Emil qui restent ne sont pas plus épargnés que sa chaîne de baptême. Lilly affirme certes dans un premier temps face à son mari Hannes l'avoir mise dans la tombe d'Emil, mais elle avoue ensuite l'avoir échangée contre du pain, des tranches de saucisson, deux pommes et deux mandarines. La recherche de nourriture est la première préoccupation des personnages qui vivent cette fin de guerre. On en trouve parfois dans les maisons bombardées, sous le stuc des plafonds écroulés ou dans les magasins éventrés comme dans celui des frères Barrasch dans le chapitre consacré à Halka, la mère de Boris. Sur le tard, Eustachius est

18. - www.der-siebte-sprung.de/memories-cascading/

19. - Ulrike Draesner: Sieben Sprünge vom Rand der Welt, op. cit., p. 129.

20. - «Mit knurrendem Magen» (Ibid., p. 98).

21. - „Warten, Zug fahren, hungern, zu Fuß gehen, warten, frieren“ (Ibid., p. 172). 
encore marqué par l'angoisse de la faim qu'il a connue lors de la fuite de Silésie et dans des sortes de visions il voit une scène où les fenêtres sont barricadées, où « tous semblaient vieux et gris et se nourrissaient de pain rassis $\gg^{22}$.

En temps de guerre, la table ne sert plus obligatoirement à partager les repas, mais est détournée de son usage, comme nous le montre le chapitre consacré à Eustachius où celui-ci raconte devoir dormir sur la table, car le lit a été brûlé pour se chauffer. Plus tard, c'est au tour de la table d'être brûlée et l'on mange alors sur une valise. Ou bien, on met sur la table que l'on a trouvée dans les maisons abandonnées, en apparence sans s'émouvoir, les nappes et la vaisselle des personnes qui avant nous ont occupé la maison et ont dû, suite à l'évacuation de la ville, fuir. La table est ici un endroit où l'on rêve du passé perdu, comme Grazyna, la grand-mère de Boris, qui, dans ses pensées, est à table toujours dans la ville de Lviv (Lemberg) qu'elle a dû quitter. La cuisine devient alors un lien privilégié pour tenter de retrouver ce passé perdu. Dans le chapitre qui fait intervenir Halka, on voit sa mère Grazyna, cuisiner sans relâche des plats de sa région natale qu'elle décore, avec amour, des monuments de la ville confectionnés avec de la pâte :

Grazyna hingegen verließ die Wohnung immer seltener, kochte und buk immer mehr. Jeden dritten Tag tischte sie nun selbstgemachte Piroggen auf [...]. Je länger wir blieben, umso ostpolnischer wurde ihre Küche, am Morgen ging Hefeteig in der Schüssel, am Abend der nächste [...]. Die Hefepasteten füllte sie mit Resten: Fleisch, Fisch, Reis, Gemüse, hartgekochte Eier, Mamuś wurde erfinderisch und setzte Teiggitter obenauf, in denen man mit etwas Mühe den Turm der Lemberger Kathedrale oder die Umrisse des Opernhauses erkennen konnte, und dann stand sie in der Küche und $\mathrm{a} ß$ das Kunstwerk allein, da Vater und ich in Kulebiak-Streik getreten waren $[\ldots]^{23}$.

Lorsque, pendant le repas africain, Simone Grolmann entend parler Boris Nienalt des conséquences d'événements traumatisants sur les générations suivantes, elle se demande tout d'abord, en être rationnel, comment l'expliquer physiologiquement. Mais les propos de Boris ont fait mouche et elle voudra en savoir plus. Elle voudra aussi lui être plus proche physiquement. On la voit ainsi changer de place et se mettre du côté de la table où est assis Boris. Ils sont alors si près l'un de l'autre que leurs bras s'effleurent et ils savourent tous les deux cette " proximité entre étrangers qui se sont raconté quelque chose d'intime sur leur

22. - «[... alle sähen alt und grau aus und ernährten sich von trockenem Brot» (Ibid., p. 544).

23. - Ibid., p. 459. 
vie $»^{24}$, avant d'être interrompus par le téléphone portable de Simone qui les ramène au monde qui les entoure. Ils n'avaient même pas remarqué que le restaurant s'était vidé et que les propriétaires étaient déjà en train de ranger, tant ils étaient absorbés par leurs confidences.

Plus tard, dans le chapitre $n^{\circ} 5$ qui lui est à nouveau consacré, Simone se rendant à Kreisau sur le lieu du séminaire de Boris, revient en pensée sur le repas africain et se remémore ce qui lui a plu : la voix de Boris bien sûr, ses taquineries, son odeur, mais aussi son sérieux et leur connivence :

Er stellte Fragen, die mir noch niemand gestellt hatte, und ich erzählte ihm Dinge, die ich ohne ihn nicht gewusst hätte 25 .

Sous le choc de l'émotion, elle raconte ici avoir eu, après le repas africain, une vision déterminante, celle d'un « futur inexplicable $»^{26}:$ la vision du visage de Boris dans vingt ans. De retour de Kreisau, le lecteur retrouve Simone à table, dans des circonstances bien différentes, cette fois-ci en compagnie de sa fille Esther et de son mari Johnny. L'attitude de Johnny est révélatrice de leur relation à la dérive. Il s'assoit au bout de la table, «très loin »27, et Simone est alors forcée de constater la fin imminente de leur couple :

Für Augenblicke sah ich uns am Tisch sitzen wie auf einer Bühne, komisch, grotesk, überdeutlich. Ich sah, dass wir dabei waren, voneinander als Familie Abschied zu nehmen ${ }^{28}$.

Le repas africain ne tient que sur quelques pages dans un roman qui en compte 555, ne constitue qu'un court épisode dans un livre qu'Ulrike Draesner a mis huit ans à écrire. Dans ce "kaléidoscope des souvenirs $»^{29}$, le repas africain présente toutefois certaines idées maîtresses du roman, les questions se trouvant au cœur même du roman étant, selon l'auteur, la nécessité de comprendre comment « les migrations forcées et la violence » agissent sur l'être humain, comment les « paysages psychiques » se transmettent d'une génération à l'autre. Ce repas constitue le début de la relation amoureuse entre Boris et Simone qui n'est cependant qu'ébauchée dans le roman. Seules ça et là, quelques remarques montrent au fil de l'œuvre que Simone et Boris forment à présent un couple. Suite au repas africain, Boris sera celui qui reprendra contact

24. - «Eine besondere Nähe umfing uns, die Nähe zwischen Fremden, die sich etwas Intimes aus ihren Leben erzählen» (Ibid., p. 131).

25. - Ibid., p. 253.

26. - «[...] unerfindlichen Zukunft $[\ldots] »($ Ibid.).

27. - «Johnny setzte sich ans Kopfende des Tisches. Weit weg» (Ibid., p. 275).

28. - Ibid., p. 276.

29. - www.der-siebte-sprung.de/der-roman. 
avec Simone. Après avoir eu une envie irrépressible de gâteaux sucrés, de gâteaux tels qu'en mangeaient, à Wrocław, Madame Waleńczak et Madame Kuriata à la bibliothèque où Boris passait son temps, Boris voit subitement clair dans la relation qu'il entretient avec Simone et qu'il veut à présent poursuivre. Il lui parle au téléphone sans relâche du vieux Wrocław, de la nostalgie de ces femmes qui avaient quitté la Pologne orientale, de leur tristesse de ne plus jamais pouvoir retrouver les mêmes gâteaux de pâte sablée, les mêmes gâteaux au pavot, de la force du souvenir et de la nostalgie qui menace le présent et peut l'occulter, des dégâts sur les générations d'après, tout en sachant qu'il veut à présent retrouver Simone et la garder. 
